\title{
Article \\ Performance Analysis of Heterogeneous Networks in presence of Deliberate Jammers using Reverse Frequency Allocation
}

\author{
Muhammad Ihsan Ur Rehman ${ }^{1}$,Muhammad Qasim ${ }^{2}{ }_{\text {, Abdul wakeel }}^{3}{ }^{3}$, Mehmood Alam $^{4}$, Mir Yasir \\ Umair 5 \\ 1 Department of Electrical Engineering, Military College of Signals(MCS), National University of Sciences and \\ Technology(NUST),Adiala Road, Lalazar, Rawalpindi,46000, Pakistan; ehsan.rehman333@gmail.com \\ 2 Department of Electrical Engineering, Military College of Signals(MCS) , National University of Sciences \\ and Technology(NUST),Adiala Road, Lalazar, Rawalpindi,46000, Pakistan; mqasim4@gmail.com \\ 3 Department of Electrical Engineering, Military College of Signals(MCS), National University of Sciences \\ and Technology(NUST),Adiala Road, Lalazar, Rawalpindi,46000, Pakistan; awakeel@mcs.edu.pk \\ 4 Department of Electrical Engineering , Military College of Signals(MCS) , National University of Sciences \\ and Technology(NUST),Adiala Road, Lalazar, Rawalpindi,46000, Pakistan; mehmood.alam@mcs.edu.pk \\ 5 Department of Electrical Engineering, Military College of Signals(MCS) , National University of Sciences \\ and Technology(NUST),Adiala Road, Lalazar, Rawalpindi,46000, Pakistan; miryasir@mcs.edu.pk
}

Version September 2, 2021 submitted to Journal Not Specified

\begin{abstract}
The demand of high data rate and ubiquitous coverage in heterogeneous cellular (HetNets) is increasing progressively. In order to meet this demand, sophisticated model having applied interference reduction scheme and cell association technique is needed. The small base station (sBS are deployed inside the broadcasting area of macro base station (mBS), in heterogeneous cellular networks (HetNets). Since mBS has high transmission power therefore a large number of users get connected with mBS. This causes disproportion of load distribution across the HetNets. For load balancing users from high power mBS are migrated to low power sBS to increase network capacity and to decrease the load from mBS. This results in interference in the communication signal because of strong mBS Interference. Hence, we need interference management technique to mitigate interference and user association and to efficiently use sBSs' resources. Inter-cell interference (ICI) limit the HetNets' performance. Additionally, there exist deliberate jamming interference which depends on jammers transmission power and its proximity with the target, which notably degrades the network performance. In this paper, we employ reverse frequency allocation scheme (RFA) to reduce inter cell interference, deliberate jamming interference and to accomplish load balancing. The proposed setup is analyzed inquisitively and with the help of simulations. The result shows reduction in interferences as well as balance of load distribution in the network achieved by employing RFA scheme together with cell association.
\end{abstract}

Keywords: Heterogeneous Cellular Networks,Deliberate Jamming, Inter-cell Interference, Independent Homogenous Poisson Point Process, Coverage Probability, Reverse Frequency Allocation

\section{Introduction}

\subsection{Motivation}

As the number of cellular users are increasing, hence the need of higher bandwidth, higher capacity and ubiquitous coverage is also increasing. Therefore, wireless data networks are slowly 

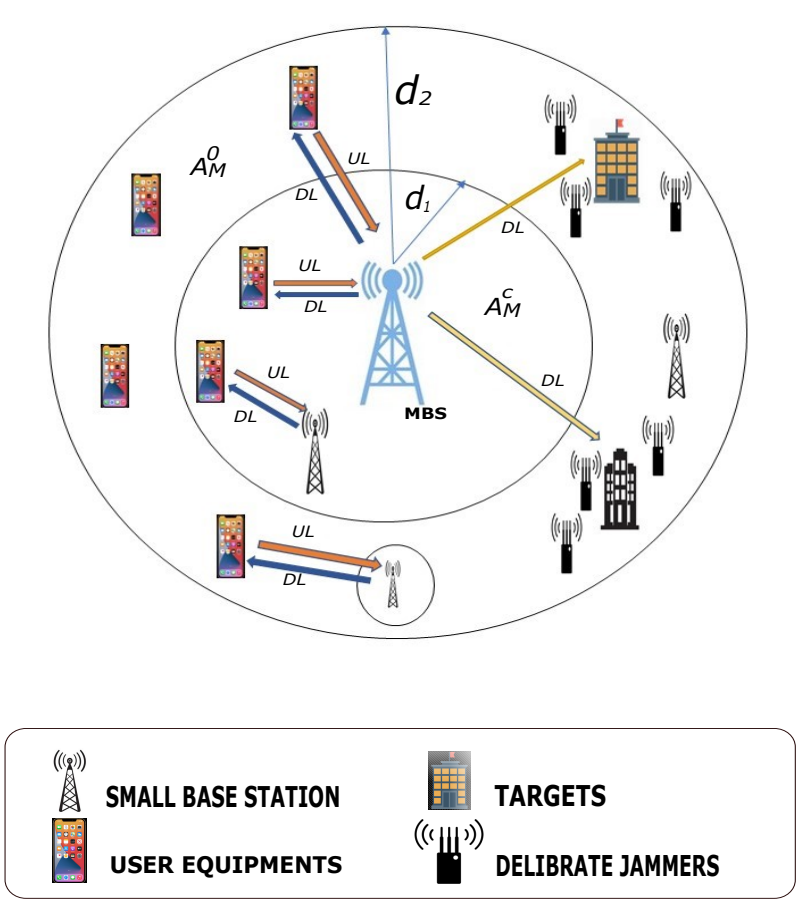

Figure 1. The suggested two tier HetNet System Model with RFA and Deliberate jamming.

evolving to adjoin the requirements [1]. In modern times deliberate jamming (DJ) is also an issue. It can be used for distributed denial of service attack in cellular networks. For such purpose, the assailant must have information about the networks characteristics such as transmission power duration and position. So that with the help of this information legitimate communication of network can be disrupted.

The suggested work investigates the uplink broadcasting recital of HetNets in ubiquity of both deliberate jamming interference and (ICI). To reduce deliberate jamming interference and ICI, reverse frequency allocation is implemented that is a dynamic interference receding strategy.

To facsimile the locations of base stations, Poisson point process (PPP) has been suggested because of its precision and complaisant. The positions of users and macro base stations (mBSs) are marked by using independent homogeneous Poisson Point Process (IHPPP).

Since orthogonal frequency Division multiplexing (OFDM) is used in (HetNets) therefore system experience inconsequential intra cell interference however ICI persists the leading factor restricting the performance [2,3]. In Non Uniform HetNets (NU-HetNets) sBSs positioned by using Poisson Hole process while users and mBSs are positioned using (IHPPP). NUHetNets ameliorate the performance of network coverage by alleviating ICI [4].

Many interference alleviating setups, for instance soft frequency reuse (SFR)[5] and fractinal frequency reuse (FFR)[6], were researched. Since SFR system employs frequency reuse therefore it yields higher spectral efficiency and while FFR system employs partitioning [7] a usable bandwidth therefore it yields reduced interference. Reverse frequency allocation $[2,6]$ which is a dynamic resource management system accessible at avant-garde. RFA make utmost bandwidth in a cell utilizable for mBS as well as sBS. Therefore, RFA has better spectral efficiency than SFR and FFR. The suggested work will implement RFA in HetNets to alleviate ICI and deliberate jamming interference therefore improving uplink (UL) broadcasting recital in HetNets. This suggested network composition by alleviating ICI and deliberate jamming interference in HetNets yields higher network capacity 
Table 1. List of abbreviations

\begin{tabular}{ll}
\hline Abbreviations & Description \\
\hline HetNet & Heterogeneous Cellular Network \\
IJs & Deliberate Jammers \\
DJs-I & Deliberate Jammers Interference \\
ICI & Inter Cell Interference \\
sBS & Small Base Station \\
UL & Uplink \\
mBSs & Macro Base Stations \\
RFA & Reverse Frequency Allocation \\
U-HetNet & Uniform Heterogeneous Cellular Network \\
NU-HetNet & Non Uniform Heterogeneous Cellular Network \\
FFR & Fractional Frequency Reuse \\
SFR & Soft Frequency Reuse \\
DL & Downlink \\
M-EUs & MBS edge-users \\
SIR & Signal-to-interference ratio \\
LT & Laplace transform \\
IHPPP & Independent Homogeneous Poisson Point Process \\
\hline
\end{tabular}

\subsubsection{Proposed Work}

This suggested paper implements RFA in HetNets to alleviate DJs-I and ICI to enhance the broadcasting recital in HetNets. This network framework yields higher network capacity by efficiently alleviating DJs-I and ICI in HetNets. The used index of abbreviations in Table 1 and notations in Table 2 is given as:

\subsection{Related Work}

In [8] the author have studied different kinds of jamming attacks these jammers such as equalization jammers, automatic gain control jammers, wide band jammers and partial band jammers have been thoroughly studied . In [9], the authors have studied multiple input and multiple output (MIMO) networks in residence of advanced jamming attacks. The results indicate that recital mortification in network recital because of jammers transmission power increment. The mBS coverage area has been splitted into two sub areas that is outer cell area and inner cell area in [10] for the better analysis of coverage probability in these areas. The users migrating in inner cell area faces severe interference because of proximity to mBS and the users located in outer cell area experience low SINR because of their far-flung position [10].Since mBS has strong signal transmission therefore sBSs situated in the inner cell area of mBS have truncated broadcasting region [10].

To utilize RFA in HetNets, two nonintersecting areas have been modeled from broadcasting area of mBs having inner and outer areas [10,11].The author in [10] implements RFA along with load balancing to reduce ICI. The outcome suggest notable broadcasting recital boost for mBS edge user. The authors have studied NU-HetNets accompanied by SFR in [9]. Authors have derived coverage probability expressions for U-HetNets as well as NU-HetNets. The result indicate notable boost in broadcasting area because of implicit reduction in ICI.

One of the interference reduction procedures includes fractional frequency reuse (FFR) [12], in which the whole available spectrum is partitioned into multiple sub spectra for reduction in ICI and to boost broadcasting recital. This method is ineffective because of partitioning of spectrum. The authors have proposed another procedure of Soft Fractional frequency reuse in [13] that is more coherent technique than FFR.

To utilize RFA in HetNets and its worthwhile recital assessment two non-intersecting areas have 
Table 2. Symbols summary

\begin{tabular}{|c|c|}
\hline Symbols & Explaination \\
\hline$\varphi_{\mathrm{S}}, \varphi_{\mathrm{M}}, \varphi_{u}, \varphi_{J}$ & $\begin{array}{l}\text { IHPPPs of sBSs, mBSs, users, } \\
\text { and DJs, }\end{array}$ \\
\hline$\beta$ & $\begin{array}{l}\text { The path loss exponent } \\
\forall \beta_{\mathrm{M}}=\beta_{\mathrm{S}}=\beta \text {, and } \beta>2\end{array}$ \\
\hline$d_{1}, d_{2}$ & $\begin{array}{l}\text { The radius of } A_{\mathrm{M}}^{c} \text { and } A_{\mathrm{M}}^{o} \\
\text { respectively }\end{array}$ \\
\hline$\varphi_{\mathrm{s}}, \varphi_{\mathrm{m}}$ and $\varphi_{j}$ & $\begin{array}{l}\text { The densities of sBSs, mBSs } \\
\text { and IJs }\end{array}$ \\
\hline$\kappa_{\mathrm{M}}$ & SIR threshold \\
\hline$v$ & Classical end User \\
\hline * & Represents RFA deployment \\
\hline$\gamma_{\circ}$ & Ratio of $P_{t, \mathrm{M}}$ and $P_{t, v}^{\mathrm{UL}}$ \\
\hline$\gamma_{1}$ & Ratio of $P_{t, \mathrm{~S}}$ and $P_{t, v}^{\mathrm{U}, \mathrm{v}}$ \\
\hline$\gamma_{2}$ & Ratio of $P_{t, J}$ and $P_{t, v}$ \\
\hline$\gamma_{3}$ & Ratio of $P_{t, \mathrm{~S}}^{\mathrm{D} \mathrm{L}}$ and $P_{t, v} \mathrm{UL}$ \\
\hline
\end{tabular}

been established from broadcasting area of mBS, having central area, $A_{k^{\prime}}^{c}$, and outward region, $A_{k^{\prime}}^{o}$ $\forall k \in\{\mathrm{M}, \mathrm{S}\}[14,15]$. The writers in [15], have utilized load balancing with RFA to alleviate ICI.By using the suggested setup, the results indicates the significant improvement in coverage performance for MBS edge user.

This suggested work differ in these ways:

1. In $[7,16]$ authors have investigated disparate jamming attacks in disparate networks; however, their study lacks in assessment of deliberate jamming in HetNets. Hence deliberate jamming in HetNets has been discussed in this work.

2. In $[4,5,8,10,17]$ the authors have explored RFA in HetNets and reduction of ICI. However their study lack lacks in assessment of RFA in reduction of deliberate jamming interference. Hence RFA is discussed in this work for the reduction of ICI and deliberate jamming interference.

3. In $[4,17,18]$ downlink coverage has been investigated while this work focuses on analysis of factors that affect uplink coverage of mBS edge users.

\subsection{Contributions and Objectives}

This paper contributes in the following areas:

1. Study of interference originated by deliberate jamming in HetNets.

2. Use of RFA scheme to reduce ICI and deliberate jamming interference for enhancement of network recital gain.

3. Analysis of coverage probability and study of SINR threshold, signal to noise ratio SNR, mBS and sBS densities.

4. Study of Uplink coverage.

5. Study of RFA scheme with simulation scenarios.

The rest of the work is categorized in the following: Sec. 2 follows about system representation. The coverage probabilities derivations have been studied in Sec. 3. Sec. 4 discusses the results of the suggested system. The last Sec. 5, finishes the paper.

\section{System Model}

We will be implemented RFA while considering the deployment of sBS in the broadcasting region of mBS. Moreover interference mitigation in network layout schemes have been introduced along with 


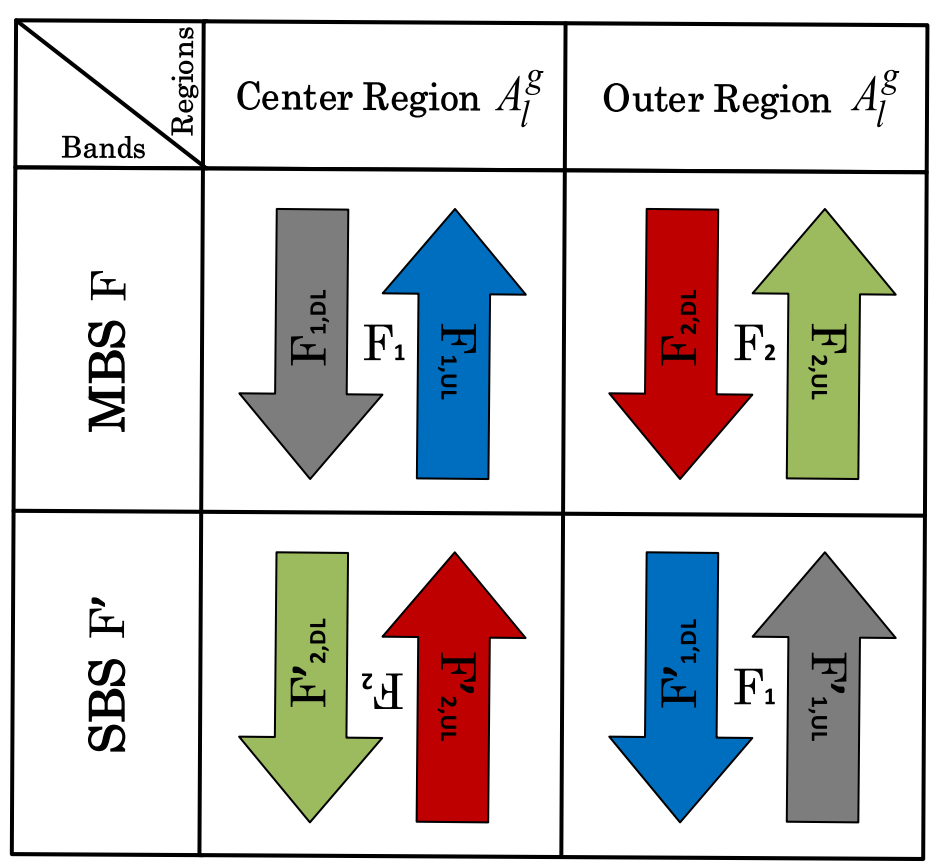

Figure 2. The two-tier HetNet representation along with RFA.

deliberate jamming mechanism. Moreover, mathematical preliminaries which are acquired here, are also utilized in Sec. 3 for derivation of coverage probabilities.

\subsection{Network Layout and Assumptions}

HetNets facsimile having two levels has been proposed having users, DJs, sBSs and mBSs. The users, DJs,sBSs and mBSs are modeled via IHPPPs with densities $\varphi_{\mathrm{u}}, \varphi_{\mathrm{J}}, \varphi_{\mathrm{S}}$ and $\varphi_{\mathrm{M}}$, respectively.In the legitimate communication band DJs disseminate undesirable energies which abase network recital. .For the reduction of DJs-I and ICI, a dynamic interference reduction method RFA is enlisted. For the review, a classic user is supposed . $\beta$ indicates path loss constant while $|h|$ represents Rayleigh fading gain. User interrelation has been achieved by using maximum power scheme technique [19].

\subsection{Deliberate Jamming Apparatus}

Deliberate jammers (D-js) introduce interference in the permissible transmissions and thus reduced network coverage[7]. D-js distributed randomly using IHPPP in the mBS broadcasting area. Since $\mathrm{mBS}$ edge users are located at a great distance from $\mathrm{mBS}$, thus experience critical degradation in network performance also ICI is a network degrading element. Thus D-js jam desired communication frequency. By increasing, D-js and their transmission range, UL of M-EUs in HetNets can entirely be jammed.

\subsection{Use of Reverse Frequency Allocation}

In SFR, the frequency band is further partitioned in two sub-bands. One of the sub band is assigned to the users and sBSs in mBS that located in center region and the second sub band assigned to SBS and the users in the outer region [5]. RFA is the advanced form of SFR having enhanced broadcast recital[20].In RFA frequency sub band are additionally divided into uplink and downlink frequency as compared to SFR. This permits notable interference mitigation as compared to SFR $[17,20]$. The radio relay direction of sBS are allocated in reverse direction as compared to $\mathrm{mBS}$.The mBS DL frequencies are assigned to the UL of sBSs, while mBS UL frequencies are assigned to the DL of sBSs [21].The RFA significantly increases the network capacity of the mBS coverage area when this 
form of frequency allocation apparatus is used. As a result, RFA efficiently reduced the interference in this way and improves spectral efficiency as well as network coverage.The allocation of frequency resources is perfect, resulting in less co-tier and cross-tier interference and a higher data rate. [22].

In RFA no predetermined spectrum is allotted to sBS therefore it mitigates interference and increases coverage area. By introducing RFA the complete spectrum of $\mathrm{mBS}$ is made accessible to sBSs in non-intersecting areas but in reverse direction.

From the Fig. 2, For RFA, the sub bands are in reverse order used in mBSs and sBSs that is $A_{l}^{g}$ $\forall l \in(\mathrm{M}, \mathrm{S})$ and $g \in(c, o)[23]$.

For the implementation of RFA, the allotted frequency $\mathrm{F}$, is further divided in two sub bands, $\mathrm{F}_{1}$ and $F_{2}$, such that $F=\bigcup_{z \in(1,2)} F z$, as represented in Fig. 2. F1 and F2 are additionally partitioned in UL and DL sub-carriers. These sub bands are categorized as $\mathrm{F}_{1}=\mathrm{F}_{1, \mathrm{UL}}+\mathrm{F}_{1, \mathrm{DL}}$ and $\mathrm{F}_{2}=\mathrm{F}_{2, \mathrm{UL}}+\mathrm{F}_{2, \mathrm{DL}}$.

\section{Analysis of Coverage Probability}

Since $v$ is situated in $A_{\mathrm{M}}^{c}$ and $A_{\mathrm{M}}^{o}$ so here in this segment expression of coverage probabilities for cited below network frameworks are extricated.

1. Evaluation of UL in case of DJs without RFA in terms of coverage probabilities.

2. Evaluation of UL in case of DJs with RFA in terms of coverage probabilities.

\subsection{Evaluation of UL in case of DJs without RFA in terms of coverage probabilities}

For the disruption of uplink communication of M-EU in HetNets, DJ-s are positioned identically around $\mathrm{mBS}$ broadcasting region by using IHPPP. The limiting factors of efficiency in such networks are DJS-I and ICI. Thus the equation for uplink coverage probability, $P_{A_{\mathrm{M}}^{\mathrm{L}}}^{\mathrm{UL}}\left(\kappa_{\mathrm{M}}\right)$, for mBS accompanying $v$ in $A_{\mathrm{M}}^{c}$ without RFA and in the presence of DJ-s can be given as

$$
P_{A_{\mathrm{M}}^{\mathrm{L}}}^{\mathrm{UL}}\left(\kappa_{\mathrm{M}}\right)=P\left(\operatorname{SIR}_{\mathrm{M}}^{\mathrm{UL}}>\kappa_{\mathrm{M}}\right) \text {. }
$$

Here, $\kappa_{M}$ is UL SIR threshold.Moreover $\operatorname{SIR}_{M}^{U L}$ represents the received UL SIR. SIR $\mathrm{M}_{\mathrm{U}}^{\mathrm{L}}$ from equation (1) it can be found as

$$
\begin{gathered}
\operatorname{SIR}_{\mathrm{M}}^{\mathrm{UL}}=\frac{P_{t, v}^{\mathrm{UL}}\left|h_{\mathrm{M}}\right|^{2} r_{\mathrm{M}}^{-\beta}}{I_{\mathrm{M}, A}+I_{\mathrm{S}, A}+I_{J, A}}, \\
=\frac{P_{t, v}^{\mathrm{UL}}\left|h_{\mathrm{M}}\right|^{2} r_{\mathrm{M}}^{-\beta}}{\sum_{l \in \phi_{\mathrm{M}}} P_{t, l}\left|h_{l}\right|^{2} r_{l}^{-\beta}+\sum_{k \in \phi_{\mathrm{S}}} P_{t, k}\left|h_{k}\right|^{2} r_{k}^{-\beta}+\sum_{j \in \phi_{J}} P_{t, j}\left|h_{j}\right|^{2} r_{j}^{-\beta}} .
\end{gathered}
$$

In eq(2), the interference of $\mathrm{UL}$ in $A_{\mathrm{M}}^{c}$ is the total number of mBS-tier interferences. $I_{\mathrm{M}, A}, \mathrm{sBS}$-tier $I_{\mathrm{S}, A}$, and of DJs, $I_{J, A} \cdot r_{(\cdot)}^{-\beta}$ represents the separation from either BS or DJs. However, $P_{t, v}^{\mathrm{UL}}$ represents the power transmitted in UL. While $P_{t, k}, P_{t, l}$ and $P_{t, j}$ represents the powers transmitted of sBSs, mBSs and DJs . $\varphi_{\mathrm{S}}, \phi_{\mathrm{M}}$ and $\varphi_{J}$ represents the IHPPPs of sBSs, mBSs and DJs. However, $A$ represents the mBS broadcasting region, $A=A_{\mathrm{M}}^{c} \cup A_{\mathrm{M}}^{o}$.Using (2), (1) can be written as

$$
P_{A_{\mathrm{M}}^{\mathrm{c}}}^{\mathrm{UL}}\left(\kappa_{\mathrm{M}}\right) \stackrel{(1)}{=} P\left(\frac{P_{t, v}^{\mathrm{UL}}\left|h_{\mathrm{M}}\right|^{2} r_{\mathrm{M}}^{-\beta}}{I_{\mathrm{M}, A}+I_{\mathrm{S}, A}+I_{J, A}}>\kappa_{\mathrm{M}}\right)
$$




$$
\begin{aligned}
& \stackrel{(2)}{=} \mathrm{E}_{r_{\mathrm{M}}, I_{\mathrm{M}, A}, I_{\mathrm{S}, A}, I_{J, A}}\left[\exp \left(-\frac{r_{\mathrm{M}}^{\beta} \kappa_{\mathrm{M}}}{P_{t, v}^{\mathrm{UL}}}\left(I_{\mathrm{M}, A}+I_{\mathrm{S}, A}+I_{J, A}\right)\right)\right] \\
& \stackrel{(3)}{=} \mathrm{E}_{r_{\mathrm{M}}, I_{\mathrm{M}, A}, I_{\mathrm{S}, A}, I_{J, A}}\left[\exp \left(-s\left(I_{\mathrm{M}, A}+I_{\mathrm{S}, A}+I_{J, A}\right)\right)\right] \\
& \stackrel{(4)}{=} \mathrm{E}_{r_{\mathrm{M}}}\left[\mathrm{E}_{I_{\mathrm{M}, A}} \exp \left(-s\left(I_{\mathrm{M}, A}\right)\right) \times \mathrm{E}_{I_{\mathrm{S}, A}} \exp \left(-s\left(I_{\mathrm{S}, A}\right)\right) \times \mathrm{E}_{I_{J, A}} \exp \left(-s\left(I_{J, A}\right)\right)\right] \\
& \left.\stackrel{(5)}{=} \mathrm{E}_{r_{\mathrm{M}}}\left[\mathcal{L}_{I_{\mathrm{M}, A}}(s) \times \mathcal{L}_{I_{\mathrm{S}, A}}(s) \times \mathcal{L}_{I_{J, A}}(s)\right]\right|_{s=\frac{r_{\mathrm{M}}^{\beta} \kappa_{\mathrm{M}}}{P_{t, v}^{\mathrm{UL}}}} .
\end{aligned}
$$

Here, $\mathcal{L}_{I_{\mathrm{M}, A}}(s), \mathcal{L}_{I_{\mathrm{S}, A}}(s)$, and $\mathcal{L}_{I_{J, A}}(s)$ represents the laplace transform $(\mathrm{LT})$ of $I_{\mathrm{M}, A}, I_{\mathrm{S}, A}$, and $I_{J, A}$. $\mathrm{E}[\cdot]$ represents the expectation of LTs.

From the above equation (3), step (1) is acquired by the coverage probability defination [15]. And the step (2) is acquired from the definition of void probability of independent homogenous poisson point process.Step (3) is derived by substituting the value of $s$ into step (2) where $s$ is $\frac{r_{\mathrm{M}}^{\beta} \kappa_{\mathrm{M}}}{P_{t, v}^{\mathrm{UL}}}$. However, step (4) is derived from the defination of exponential property, i.e., $\exp (a+b)=\exp (a) \times \exp (b)$. Step (5) is eventually inferred from Step (4) via the LT defination (see (2.12) of [24]).

The Laplace transform obtained by the mbs tier looks like this., $\mathcal{L}_{I_{\mathrm{M}, A}}(s)$, in $A$, is calculated

$$
\begin{aligned}
& \mathcal{L}_{I_{\mathrm{M}, A}}(s)=\exp \left(\frac{\varphi_{\mathrm{M}} \pi \gamma_{\circ} \kappa_{\mathrm{M}} d_{2}^{(2-\beta)} r_{\mathrm{M}}^{\beta}}{\beta / 2-1}{ }_{2} F_{1}\left(1,1-\frac{2}{\beta}, 2-\frac{2}{\beta},-\gamma_{\circ} \kappa_{\mathrm{M}}\left(\frac{r_{\mathrm{M}}}{d_{2}}\right)^{\beta}\right)-\right. \\
& \left.\frac{\varphi_{\mathrm{M}} \pi \gamma_{\circ} \kappa_{\mathrm{M}} y^{(2-\beta)} r_{\mathrm{M}}^{\beta}}{\beta / 2-1}{ }_{2} F_{1}\left(1,1-\frac{2}{\beta}, 2-\frac{2}{\beta},-\gamma_{\circ} \kappa_{\mathrm{M}}\left(\frac{r_{\mathrm{M}}}{y}\right)^{\beta}\right)\right) .
\end{aligned}
$$

Proof: The proof of (4)is given in Appendix A . In equation (4), $\gamma_{\circ}$ is the correlation of $P_{t, \mathrm{M}}$ and $P_{t, v}^{\mathrm{UL}}$, where $P_{t, \mathrm{M}}$ represents the power transmitted by mBS-tier.

By applying the similar method in (4), $A$ is calculated by using the interference LT obtained from the sBS, $\mathcal{L}_{I_{S, A}}(s)$

$$
\begin{aligned}
& \mathcal{L}_{I_{\mathrm{S}, A}}(s)=\exp \left(\frac{\varphi_{\mathrm{S}} \pi \gamma_{1} \kappa_{\mathrm{M}} x_{2}^{(2-\beta)} r_{\mathrm{M}}^{\beta}}{\beta / 2-1}{ }_{2} F_{1}\left(1,1-\frac{2}{\beta}, 2-\frac{2}{\beta},-\gamma_{1} \kappa_{\mathrm{M}}\left(\frac{r_{\mathrm{M}}}{x_{2}}\right)^{\beta}\right)-\right. \\
& \left.\frac{\varphi_{\mathrm{S}} \pi \gamma_{1} \kappa_{\mathrm{M}} x_{1}^{(2-\beta)} r_{\mathrm{M}}^{\beta}}{\beta / 2-1}{ }_{2} F_{1}\left(1,1-\frac{2}{\beta}, 2-\frac{2}{\beta},-\gamma_{1} \kappa_{\mathrm{M}}\left(\frac{r_{\mathrm{M}}}{x_{1}}\right)^{\beta}\right)\right) .
\end{aligned}
$$

Here, $\gamma_{1}$ is ratio of $P_{t, \mathrm{~S}}$ and $P_{t, v}^{\mathrm{UL}}$, where $P_{t, \mathrm{~S}}$ is the power transmitted by sBS.

By applying the similar technique of (4), the interference LT obtained from the DJs, $\mathcal{L}_{I_{J}, A}(s)$, in $A$, can be represented as follows

$$
\begin{aligned}
& \mathcal{L}_{I_{J, A}}(s)=\exp \left(\frac{\varphi_{J} \pi \gamma_{2} \kappa_{\mathrm{M}} z_{2}^{(2-\beta)} r_{\mathrm{M}}^{\beta}}{\beta / 2-1}{ }_{2} F_{1}\left(1,1-\frac{2}{\beta}, 2-\frac{2}{\beta},-\gamma_{2} \kappa_{\mathrm{M}}\left(\frac{r_{\mathrm{M}}}{z_{2}}\right)^{\beta}\right)-\right. \\
& \left.\frac{\varphi_{J} \pi \gamma_{2} \kappa_{\mathrm{M}} z_{1}^{(2-\beta)} r_{\mathrm{M}}^{\beta}}{\beta / 2-1}{ }_{2} F_{1}\left(1,1-\frac{2}{\beta}, 2-\frac{2}{\beta},-\gamma_{2} \kappa_{\mathrm{M}}\left(\frac{r_{\mathrm{M}}}{z_{1}}\right)^{\beta}\right)\right) .
\end{aligned}
$$

Here, $\gamma_{2}$ is the ratio of $P_{t, J}$ and $P_{t, v}^{\mathrm{UL}}$ where $P_{t, J}$ is transmitted power of DJs and $z_{1}$ and $z_{2}$ represents the jamming areas of the jammers, s.t., $z_{1} \leq z_{2}$. 


$$
\begin{aligned}
& P_{A_{\mathrm{M}}^{c}}^{\mathrm{UL}}\left(\kappa_{\mathrm{M}}\right)=\frac{2 \pi \varphi_{\mathrm{M}}}{1-\exp \left(-\varphi_{\mathrm{M}} \pi d_{1}^{2}\right)} \int_{y}^{d_{1}} \exp \left(\frac { \pi \kappa _ { \mathrm { M } } r _ { \mathrm { M } } ^ { \beta } } { \beta / 2 - 1 } \left[\varphi_{\mathrm{M}} \gamma_{\circ} d_{2}^{(2-\beta)} \mathcal{J}\left(\beta,-\kappa_{\mathrm{M}} \gamma_{\circ}\left(\frac{r_{\mathrm{M}}}{d_{2}}\right)^{\beta}\right)-\varphi_{\mathrm{M}} \gamma_{\circ} y^{(2-\beta)} \mathcal{J}\left(\beta,-\kappa_{\mathrm{M}} \gamma_{\circ}\left(\frac{r_{\mathrm{M}}}{y}\right)^{\beta}\right)+\right.\right. \\
& \varphi_{\mathrm{S}} \gamma_{1} x_{2}^{(2-\beta)} \mathcal{J}\left(\beta,-\kappa_{\mathrm{M}} \gamma_{1}\left(\frac{r_{\mathrm{M}}}{x_{2}}\right)^{\beta}\right)-\varphi_{\mathrm{S}} \gamma_{1} x_{1}^{(2-\beta)} \mathcal{J}\left(\beta,-\kappa_{\mathrm{M}} \gamma_{1}\left(\frac{r_{\mathrm{M}}}{x_{1}}\right)^{\beta}\right)+\varphi_{J} \gamma_{2} z_{2}^{(2-\beta)} \mathcal{J}\left(\beta,-\kappa_{\mathrm{M}} \gamma_{2}\left(\frac{r_{\mathrm{M}}}{z_{2}}\right)^{\beta}\right)- \\
& \left.\left.\varphi_{J} \gamma_{2} z_{1}^{(2-\beta)} \mathcal{J}\left(\beta,-\kappa_{\mathrm{M}} \gamma_{2}\left(\frac{r_{\mathrm{M}}}{z_{1}}\right)^{\beta}\right)\right]-\varphi_{\mathrm{M}} \pi r_{\mathrm{M}}^{2}\right) r_{\mathrm{M}} d r_{\mathrm{M}} \\
& P_{A_{\mathrm{M}}^{o}}^{\mathrm{UL}}\left(\kappa_{\mathrm{M}}\right)=\frac{2 \pi \varphi_{\mathrm{M}}}{\exp \left(-\varphi_{\mathrm{M}} \pi d_{1}^{2}\right)} \int_{d_{1}}^{d_{2}} \exp \left(\frac { \pi \kappa _ { \mathrm { M } } r _ { \mathrm { M } } ^ { \beta } } { \beta / 2 - 1 } \left[\varphi_{\mathrm{M}} \gamma_{\circ} d_{2}^{(2-\beta)} \mathcal{J}\left(\beta,-\kappa_{\mathrm{M}} \gamma_{\circ}\left(\frac{r_{\mathrm{M}}}{d_{2}}\right)^{\beta}\right)-\varphi_{\mathrm{M}} \gamma_{\circ} y^{(2-\beta)} \mathcal{J}\left(\beta,-\kappa_{\mathrm{M}} \gamma_{\circ}\left(\frac{r_{\mathrm{M}}}{y}\right)^{\beta}\right)\right.\right. \\
& +\varphi_{\mathrm{S}} \gamma_{1} x_{2}^{(2-\beta)} \mathcal{J}\left(\beta,-\kappa_{\mathrm{M}} \gamma_{1}\left(\frac{r_{\mathrm{M}}}{x_{2}}\right)^{\beta}\right)-\varphi_{\mathrm{S}} \gamma_{1} x_{1}^{(2-\beta)} \mathcal{J}\left(\beta,-\kappa_{\mathrm{M}} \gamma_{1}\left(\frac{r_{\mathrm{M}}}{x_{1}}\right)^{\beta}\right)+\varphi_{J} \gamma_{2} z_{2}^{(2-\beta)} \mathcal{J}\left(\beta,-\kappa_{\mathrm{M}} \gamma_{2}\left(\frac{r_{\mathrm{M}}}{z_{2}}\right)^{\beta}\right)- \\
& \left.\left.\varphi_{J} \gamma_{2} z_{1}^{(2-\beta)} \mathcal{J}\left(\beta,-\kappa_{\mathrm{M}} \gamma_{2}\left(\frac{r_{\mathrm{M}}}{z_{1}}\right)^{\beta}\right)\right]-\varphi_{\mathrm{M}} \pi r_{\mathrm{M}}^{2}\right) r_{\mathrm{M}} d r_{\mathrm{M}}
\end{aligned}
$$

$v$ is situated in $A_{\mathrm{M}}^{c}$ or in $A_{\mathrm{M}}^{o}$ (represented as $v_{A_{\mathrm{M}}^{c}}$ and $v_{A_{\mathrm{M}}^{o}}$, respectively), whereas corresponding the distance of $\mathrm{mBS} r_{\mathrm{M}, v}$, having PDFs of distances as (7) and (8) [25] [20]

$$
f_{r_{\mathrm{M}} \mid v_{A_{\mathrm{M}}^{c}}}\left(r_{\mathrm{M}}\right)=\frac{2 \pi \varphi_{\mathrm{M}} r_{\mathrm{M}} \exp \left(-\varphi_{\mathrm{M}} \pi r_{\mathrm{M}}^{2}\right)}{1-\exp \left(-\varphi_{\mathrm{M}} \pi d_{1}^{2}\right)},
$$

and

$$
f_{r_{\mathrm{M}} \mid v_{A_{\mathrm{M}}^{o}}}\left(r_{\mathrm{M}}\right)=\frac{2 \pi \varphi_{\mathrm{M}} r_{\mathrm{M}} \exp \left(-\varphi_{\mathrm{M}} \pi r_{\mathrm{M}}^{2}\right)}{\exp \left(-\varphi_{\mathrm{M}} \pi d_{1}^{2}\right)} .
$$

Without RFA utilization UL coverage probability expression, $P_{A_{\mathrm{M}}^{c}}^{\mathrm{UL}}\left(\kappa_{\mathrm{M}}\right)$, for MBS associated $v$ in $A_{\mathrm{M}}^{\mathrm{c}}$ whereas contemplating uniform IJs distribution, given as

$$
P_{A_{\mathrm{M}}^{c}}^{\mathrm{UL}}\left(\kappa_{\mathrm{M}}\right)=\int_{y}^{d_{1}} \mathcal{L}_{I_{\mathrm{M}, A}}(s) \times \mathcal{L}_{I_{\mathrm{S}, A}}(s) \times \mathcal{L}_{I_{J, A}}(s) f_{r_{\mathrm{M}, v} \mid v_{A_{\mathrm{M}}^{c}}}\left(r_{\mathrm{M}, v}\right) d r_{\mathrm{M}, v} .
$$

By putting values of (4), (5), (6) and (7) into (9), $P_{A_{\mathrm{M}}^{c}}^{\mathrm{UL}}\left(\kappa_{\mathrm{M}}\right)$ is written as (11).

Likewise,without RFA utilization for he uniformly distributed DJs, the UL coverage probability equation, $P_{A_{\mathrm{M}}^{o}}^{\mathrm{UL}}\left(\kappa_{\mathrm{M}}\right)$, for mBS corresponded $v$ in $A_{\mathrm{M}}^{o}$ can be obtained as

$$
P_{A_{\mathrm{M}}^{o}}^{\mathrm{UL}}\left(\kappa_{\mathrm{M}}\right)=\int_{d_{1}}^{d_{2}} \mathcal{L}_{I_{\mathrm{M}, A}}(s) \times \mathcal{L}_{I_{\mathrm{S}, A}}(s) \times \mathcal{L}_{I_{J, A}}(s) f_{r_{\mathrm{M}, v} \mid v_{A_{\mathrm{M}}^{o}}}\left(r_{\mathrm{M}, v}\right) d r_{\mathrm{M}, v} .
$$

By putting (4), (5), (6) and (8) in (10), $P_{A_{M}^{o}}^{\mathrm{UL}}\left(\kappa_{\mathrm{M}}\right)$ is written as (12).

\subsection{Evalution of UL in presence of Djs with RFA in terms of coverage probability}

The equation of UL coverage probability, $P_{A_{\mathrm{M}}^{c}}^{\mathrm{UL}, *}\left(\kappa_{\mathrm{M}}\right)$, with DJs and RFA whereas supposing $v$ in $A_{\mathrm{M}}^{c}$ is written as

$$
P_{A_{\mathrm{M}}^{c}}^{\mathrm{UL}, *}\left(\kappa_{\mathrm{M}}\right)=P\left(\mathrm{SIR}_{\mathrm{M}}^{\mathrm{UL}}>\kappa_{\mathrm{M}}\right)
$$


While deploying RFA the experienced UL interference consist of mixture of UL interference by the mBS-tier in $A_{\mathrm{M}^{\prime}}^{c}$, i.e., $I_{\phi_{\mathrm{M}}, A_{\mathrm{M}}^{c}}^{\mathrm{UL}}$,interference by the sBS-tier DL in $A_{\mathrm{M}^{o}}^{o}$, i.e., $I_{\phi_{i}, A_{\mathrm{M}}^{o}}^{\mathrm{DL}}$, and DJs interference, i.e., $I_{J, A}$. Hence, $\mathrm{SIR}_{\mathrm{M}}^{\mathrm{UL}}$ from (13) can be obtained as

$$
\mathrm{SIR}_{\mathrm{M}}^{\mathrm{UL}}=\frac{P_{t, v}^{\mathrm{UL}}\left|h_{\mathrm{M}}\right|^{2} r_{\mathrm{M}}^{-\beta}}{I_{\phi_{\mathrm{M}}, A_{\mathrm{M}}^{c}}^{\mathrm{UL}}+I_{\phi_{\mathrm{S}}, A_{\mathrm{M}}^{o}}^{\mathrm{DL}}+I_{J, A}} .
$$

Equation (14) can be expanded as

$$
\begin{aligned}
& \operatorname{SIR}_{\mathrm{M}}^{\mathrm{UL}}= \\
& \frac{P_{t, v}^{\mathrm{UL}}\left|h_{\mathrm{M}}\right|^{2} r_{\mathrm{M}}^{-\beta}}{\sum_{l \in \phi_{\mathrm{M}}} P_{t, l}^{\mathrm{UL}}\left|h_{l}\right|^{2} r_{l}^{-\beta}+\sum_{k \in \phi_{\mathrm{S}}} P_{t, k}^{\mathrm{DL}}\left|h_{k}\right|^{2} r_{k}^{-\beta}+\sum_{j \in \phi_{J}} P_{t, j}\left|h_{j}\right|^{2} r_{j}^{-\beta}} .
\end{aligned}
$$

In the above expression, the transmitted power of $v$ in mBS UL is, $P_{t, l}^{\mathrm{UL}}$, the transmitted power in SBS DL is $P_{t, k}^{\mathrm{DL}}$, and the transmitted power of IJs is $P_{t, j}$. Further more, by substituting equation (14) in equation (13), the value of $P_{A_{\mathrm{M}}^{\mathrm{L}}, *}^{\mathrm{LL}}\left(\kappa_{\mathrm{M}}\right)$ can be obtained as

$$
\begin{aligned}
& P_{A_{\mathrm{M}}^{c}, *}^{\mathrm{UL}, *}\left(\kappa_{\mathrm{M}}\right)=P\left(\frac{P_{t, v}^{\mathrm{UL}}\left|h_{\mathrm{M}}\right|^{2} r_{\mathrm{M}}^{-\beta}}{I_{\phi_{\mathrm{M}}, A_{\mathrm{M}}^{c}}^{\mathrm{UL}}+I_{\phi_{\mathrm{S}}, A_{\mathrm{M}}^{o}}^{\mathrm{DL}}+I_{J, A}}>\kappa_{\mathrm{M}}\right) \\
& =\mathrm{E}_{r_{\mathrm{M}}, I_{\phi_{\mathrm{M}}, A_{\mathrm{M}}^{c}}^{\mathrm{UL}}, I_{\phi_{\mathrm{S}}, A_{\mathrm{M}}^{o}}^{\mathrm{DL}}, I_{J, A}}\left[\exp \left(-\frac{r_{\mathrm{M}}^{\beta} \kappa_{\mathrm{M}}^{\mathrm{M}}}{P_{t, v}^{\mathrm{UL}}}\left(I_{\phi_{\mathrm{M}}, A_{\mathrm{M}}^{c}}^{\mathrm{UL}}+I_{\phi_{\mathrm{s}}, A_{\mathrm{M}}^{o}}^{\mathrm{DL}}+I_{J, A}\right)\right)\right] \\
& =\left.\mathrm{E}_{r_{\mathrm{M}}}\left[\mathcal{L}_{I_{\phi_{\mathrm{M}}, A_{\mathrm{M}}^{\mathrm{UL}}}^{\mathrm{UL}}}(s) \times \mathcal{L}_{I_{\phi_{\mathrm{S}}, A_{\mathrm{M}}^{o}}^{\mathrm{DL}}}(s) \times \mathcal{L}_{I_{J, A}}(s)\right]\right|_{s=\frac{r_{\mathrm{M}}^{\beta} \kappa_{\mathrm{M}}}{P_{t, \nu}^{\mathrm{UL}}}} .
\end{aligned}
$$

The laplace transformation of UL interference in mBS in $A_{\mathrm{M}}^{c}$, which is, $\mathcal{L}_{I_{\phi_{\mathrm{M}}}^{\mathrm{UL}}, A_{\mathrm{M}}^{c}}$, can be obtained as

$$
\begin{aligned}
& \mathcal{L}_{I_{\phi_{\mathrm{M}}, A_{\mathrm{M}}^{\mathrm{UL}}}}=\exp \left(\frac{\varphi_{\mathrm{M}} \pi \kappa_{\mathrm{M}} d_{1}^{(2-\beta)} r_{\mathrm{M}}^{\beta}}{\beta / 2-1}{ }_{2} F_{1}\left(1,1-\frac{2}{\beta}, 2-\frac{2}{\beta},-\kappa_{\mathrm{M}}\left(\frac{r_{\mathrm{M}}}{d_{1}}\right)^{\beta}\right)-\right. \\
& \left.\frac{\varphi_{\mathrm{M}} \pi \kappa_{\mathrm{M}} y^{(2-\beta)} r_{\mathrm{M}}^{\beta}}{\beta / 2-1}{ }_{2} F_{1}\left(1,1-\frac{2}{\beta}, 2-\frac{2}{\beta},-\kappa_{\mathrm{M}}\left(\frac{r_{\mathrm{M}}}{y}\right)^{\beta}\right)\right) .
\end{aligned}
$$

Proof: The proof of (17) is given in Appendix B .

However,DL interference LT of the sBS region in $A_{\mathrm{M}^{\prime}}^{o}$, i.e., $\mathcal{L}_{I_{\phi_{\mathrm{S}}, A_{\mathrm{M}}^{o}}^{\mathrm{DL}}}$, may be obtained likewise as in (17), and is written as

$$
\begin{aligned}
& \mathcal{L}_{I_{\phi_{\mathrm{S}}, A_{\mathrm{M}}^{\mathrm{DL}}}}=\mathcal{L}_{I_{\phi_{\mathrm{S}}, A_{\mathrm{M}}^{\mathrm{DL}}}}=\exp \left(\frac{\varphi_{\mathrm{S}}^{\prime} \pi \gamma_{3} \kappa_{\mathrm{M}} x_{2}^{(2-\beta)} r_{\mathrm{M}}^{\beta}}{\beta / 2-1}{ }_{2} F_{1}\left(1,1-\frac{2}{\beta}, 2-\frac{2}{\beta},-\gamma_{3} \kappa_{\mathrm{M}}\left(\frac{r_{\mathrm{M}}}{x_{2}}\right)^{\beta}\right)-\right. \\
& \left.\frac{\varphi_{\mathrm{S}}^{\prime} \pi \gamma_{3} \kappa_{\mathrm{M}} x_{1}^{(2-\beta)} r_{\mathrm{M}}^{\beta}}{\beta / 2-1}{ }_{2} F_{1}\left(1,1-\frac{2}{\beta}, 2-\frac{2}{\beta},-\gamma_{3} \kappa_{\mathrm{M}}\left(\frac{r_{\mathrm{M}}}{x_{1}}\right)^{\beta}\right)\right) .
\end{aligned}
$$




$$
\begin{aligned}
& P_{A_{\mathrm{M}}^{c}, *}^{\mathrm{UL},}\left(\kappa_{\mathrm{M}}\right)=\frac{2 \pi \varphi_{\mathrm{M}}}{1-\exp \left(-\varphi_{\mathrm{M}} \pi d_{1}^{2}\right)} \int_{y}^{d_{1}} \exp \left(\frac { \pi \kappa _ { \mathrm { M } } r _ { \mathrm { M } } ^ { \beta } } { \beta / 2 - 1 } \left[\varphi_{\mathrm{M}} d_{1}^{(2-\beta)} \mathcal{J}\left(\beta,-\kappa_{\mathrm{M}}\left(\frac{r_{\mathrm{M}}}{d_{1}}\right)^{\beta}\right)-\varphi_{\mathrm{M}} y^{(2-\beta)} \mathcal{J}\left(\beta,-\kappa_{\mathrm{M}}\left(\frac{r_{\mathrm{M}}}{y}\right)^{\beta}\right)+\right.\right. \\
& \varphi_{\mathrm{S}}^{\prime} \gamma_{3} d_{2}^{(2-\beta)} \mathcal{J}\left(\beta,-\kappa_{\mathrm{M}} \gamma_{3}\left(\frac{r_{\mathrm{M}}}{d_{2}}\right)^{\beta}\right)-\varphi_{\mathrm{S}}^{\prime} \gamma_{3} d_{1}^{(2-\beta)} \mathcal{J}\left(\beta,-\kappa_{\mathrm{M}} \gamma_{3}\left(\frac{r_{\mathrm{M}}}{d_{1}}\right)^{\beta}\right)+\varphi_{J} \gamma_{2} d_{2}^{(2-\beta)} \mathcal{J}\left(\beta,-\kappa_{\mathrm{M}} \gamma_{2}\left(\frac{r_{\mathrm{M}}}{d_{2}}\right)^{\beta}\right)- \\
& \left.\left.\varphi_{J} \gamma_{2} y^{(2-\beta)} \mathcal{J}\left(\beta,-\kappa_{\mathrm{M}} \gamma_{2}\left(\frac{r_{\mathrm{M}}}{y}\right)^{\beta}\right)\right]-\varphi_{\mathrm{M}} \pi r_{\mathrm{M}}^{2}\right) r_{\mathrm{M}} d r_{\mathrm{M}} . \\
& P_{A_{\mathrm{M}}^{0}, *}^{\mathrm{UL}, *}\left(\kappa_{\mathrm{M}}\right)=\frac{2 \pi \varphi_{\mathrm{M}}}{\exp \left(-\varphi_{\mathrm{M}} \pi d_{1}^{2}\right)} \int_{d_{1}}^{d_{2}} \exp \left(\frac { \pi \kappa _ { \mathrm { M } } r _ { \mathrm { M } } ^ { \beta } } { \beta / 2 - 1 } \left[\varphi_{\mathrm{M}} d_{2}^{(2-\beta)} \mathcal{J}\left(\beta,-\kappa_{\mathrm{M}}\left(\frac{r_{\mathrm{M}}}{d_{2}}\right)^{\beta}\right)-\varphi_{\mathrm{M}} d_{1}^{(2-\beta)} \mathcal{J}\left(\beta,-\kappa_{\mathrm{M}}\left(\frac{r_{\mathrm{M}}}{d_{1}}\right)^{\beta}\right)+\right.\right. \\
& \varphi_{\mathrm{S}}^{\prime} \gamma_{3} d_{1}^{(2-\beta)} \mathcal{J}\left(\beta,-\kappa_{\mathrm{M}} \gamma_{3}\left(\frac{r_{\mathrm{M}}}{d_{1}}\right)^{\beta}\right)-\varphi_{\mathrm{S}}^{\prime} \gamma_{3} y^{(2-\beta)} \mathcal{J}\left(\beta,-\kappa_{\mathrm{M}} \gamma_{3}\left(\frac{r_{\mathrm{M}}}{y}\right)^{\beta}\right)+\varphi_{J} \gamma_{2} d_{2}^{(2-\beta)} \mathcal{J}\left(\beta,-\kappa_{\mathrm{M}} \gamma_{2}\left(\frac{r_{\mathrm{M}}}{d_{2}}\right)^{\beta}\right)- \\
& \left.\left.\varphi_{J} \gamma_{2} y^{(2-\beta)} \mathcal{J}\left(\beta,-\kappa_{\mathrm{M}} \gamma_{2}\left(\frac{r_{\mathrm{M}}}{y}\right)^{\beta}\right)\right]-\varphi_{\mathrm{M}} \pi r_{\mathrm{M}}^{2}\right) r_{\mathrm{M}} d r_{\mathrm{M}} .
\end{aligned}
$$

Here, $\mathcal{L}_{I_{\phi_{\mathrm{S}}, A_{\mathrm{M}}^{o}}^{\mathrm{DL}}}=\mathcal{L}_{I_{\phi_{\mathrm{S}}, A_{\mathrm{M}}^{c}}^{\mathrm{DL}}}$ because $\varphi_{\mathrm{S}}$ in $A_{\mathrm{M}}^{c}$ is nearly equal to $\varphi_{\mathrm{S}}$ in $A_{\mathrm{M}}^{o} \cdot \gamma_{3}$ is ratio of $P_{t, \mathrm{~S}}^{\mathrm{DL}}$ and $P_{t, v}^{\mathrm{UL}}$ where $P_{t, \mathrm{~S}}^{\mathrm{DL}}$ is the DL power transmitted in SBSs.

By using equation (17), LT of mBS DL interference in $A_{\mathrm{M}}^{o}$, that is, $\mathcal{L}_{I_{\mathrm{M}_{\mathrm{M}}, A_{\mathrm{M}}^{o}}^{\mathrm{UL}}}$, is obtained as

$$
\begin{aligned}
& \mathcal{L}_{I_{\phi_{\mathrm{M}}^{\mathrm{U}}, A_{\mathrm{M}}^{o}}}(s)=\exp \left(\frac{\varphi_{\mathrm{M}} \pi \kappa_{\mathrm{M}} d_{2}^{(2-\beta)} r_{\mathrm{M}}^{\beta}}{\beta / 2-1}{ }_{2} F_{1}\left(1,1-\frac{2}{\beta}, 2-\frac{2}{\beta^{\prime}}-\kappa_{\mathrm{M}}\left(\frac{r_{\mathrm{M}}}{d_{2}}\right)^{\beta}\right)-\right. \\
& \left.\frac{\varphi_{\mathrm{M}} \pi \kappa_{\mathrm{M}} d_{1}^{(2-\beta)} r_{\mathrm{M}}^{\beta}}{\beta / 2-1}{ }_{2} F_{1}\left(1,1-\frac{2}{\beta}, 2-\frac{2}{\beta},-\kappa_{\mathrm{M}}\left(\frac{r_{\mathrm{M}}}{d_{1}}\right)^{\beta}\right)\right) .
\end{aligned}
$$

UL coverage probability expression, $P_{A_{\mathrm{M}}^{c}}^{\mathrm{UL}, *}\left(\kappa_{\mathrm{M}}\right)$, for correlated $v$ in mBS $A_{\mathrm{M}}^{c}$ whereas imagining uniform deployment of DJs and RFA can be obtained as

$$
P_{A_{\mathrm{M}}^{c}}^{\mathrm{UL}, *}\left(\kappa_{\mathrm{M}}\right)=\int_{y}^{d_{1}} \mathcal{L}_{I_{\phi_{\mathrm{M}}, A_{\mathrm{M}}^{c}}^{\mathrm{UL}}}(s) \times \mathcal{L}_{I_{\Phi_{\mathrm{S}}, A_{\mathrm{M}}^{\mathrm{DL}}}^{\mathrm{DL}}}(s) \times \mathcal{L}_{I_{J, A}}(s) f_{r_{\mathrm{M}, v} \mid v_{A_{\mathrm{M}}^{c}}}\left(r_{\mathrm{M}, v}\right) d r_{\mathrm{M}, v}
$$

By putting values from (6), (7), (17) and (18), in (22), $P_{A_{\mathrm{M}}^{c}}^{\mathrm{UL}, *}\left(\kappa_{\mathrm{M}}\right)$ is expressed as (20).

Therefore, UL coverage probability equation, $P_{A_{\mathrm{M}}^{\mathrm{L}}, *}^{\mathrm{LL}}\left(\kappa_{\mathrm{M}}\right)$, for $v$ correlated with $\mathrm{mBS}$ in $A_{\mathrm{M}}^{o}$, with uniform deployment of DJs and RFA, can be obtained as

$$
P_{A_{\mathrm{M}}^{o}}^{\mathrm{UL}, *}\left(\kappa_{\mathrm{M}}\right)=\int_{d_{1}}^{d_{2}} \mathcal{L}_{I_{\Phi_{\mathrm{M}}, A_{\mathrm{M}}^{o}}^{\mathrm{UL}}}(s) \times \mathcal{L}_{I_{\Phi_{\mathrm{S}}, A_{\mathrm{M}}^{\mathrm{D}}}^{\mathrm{DL}}}(s) \times \mathcal{L}_{I_{J, A}}(s) f_{r_{\mathrm{M}, v} \mid v_{A_{\mathrm{M}}^{o}}}\left(r_{\mathrm{M}, v}\right) d r_{\mathrm{M}, v} .
$$

By placing the (6), (8), (18) and (19) in (23), $P_{A_{\mathrm{M}}^{\mathrm{o}}}^{\mathrm{UL}, *}\left(\kappa_{\mathrm{M}}\right)$ is represented as (21).

\section{Results and Discussion}

We discusses the conclusions of UL coverage probability in this portion for the user $v$, assuming (i) Using RFA to cover a DJ's distribution region, the Uplink coverage against radius and (ii)Without using RFA to cover a DJ's distribution region, the Uplink coverage against radius. The results obtained 


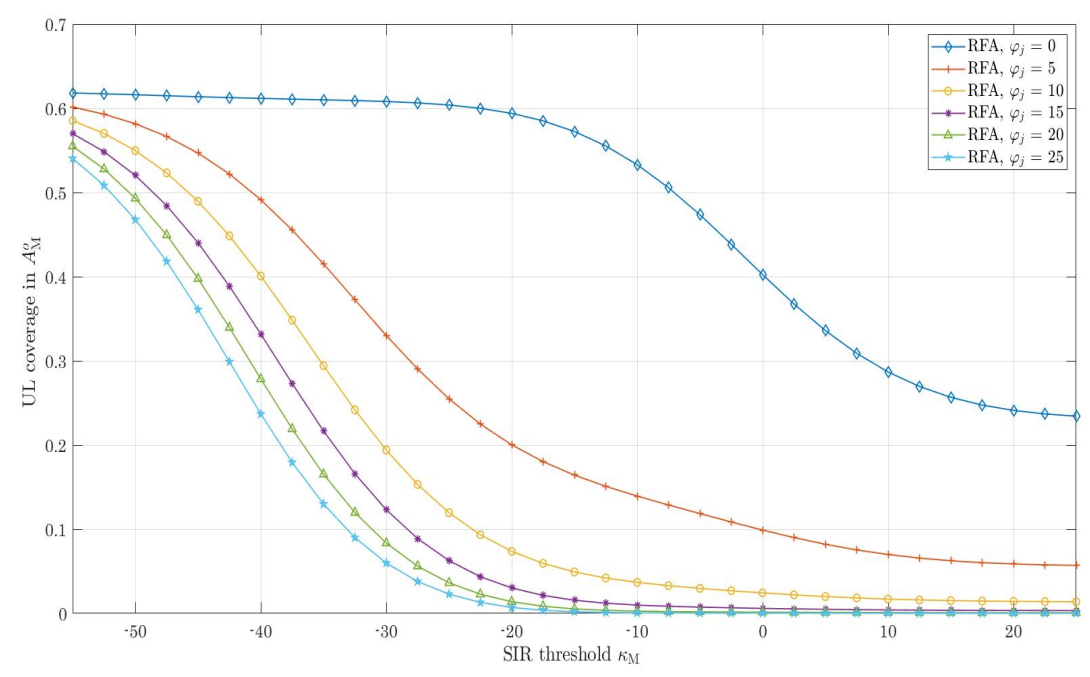

Figure 3. UL coverage versus $\kappa_{\mathrm{M}}$ and $\varphi_{J}$ in $A_{\mathrm{M}}^{o}$.

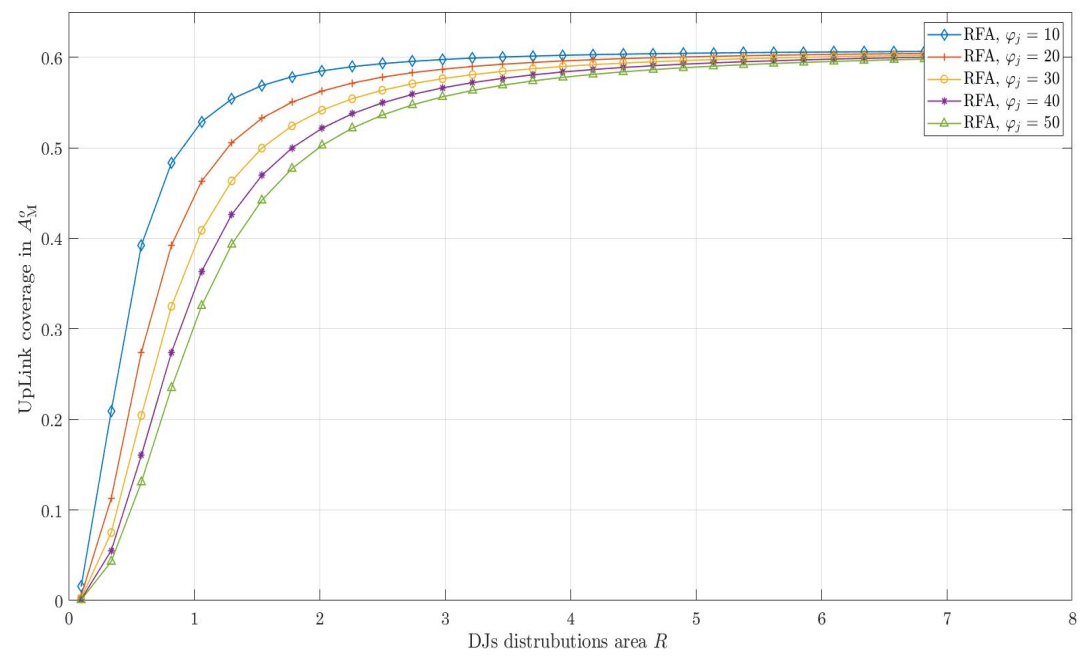

Figure 4. Using RFA to cover a DJ's distribution region, the Uplink coverage against radius .

by using the Matlab 2019b.The sBSs, mBSs and users are considered as $A=\pi(2000 \mathrm{~m})^{2}$, such that, $A=A_{\mathrm{M}}^{c} \mathrm{U} A_{\mathrm{M}}^{o}$. Additionally, the transmitted powers of sBS, mBS, DJs, and $v$ are considered as $20 \mathrm{dBm}$, $30 \mathrm{dBm}, 10 \mathrm{dBm}$, and $10 \mathrm{dBm}$, respectively. While assuming $v$ in $A_{\mathrm{M}}^{o}$ the consequences of network parameters such as $P_{t, v}^{\mathrm{UL}}, \varphi_{J}, \varphi_{\mathrm{M}}, \varphi_{\mathrm{S}}, \kappa_{\mathrm{M}}$, and $P_{t, J}$, are examined for the coverage in UL.The parameters for the simulation for the suggested network are catalogued in Table 3.

The simulated and numerical results for UL coverage probability are shown in Fig. 3 which compares different values of $A_{\mathrm{M}}^{o}$ versus $\kappa_{\mathrm{M}}$. The results indicate that higher valus of $\varphi_{J}$ causes higher DJs-I and lower UL coverage. RFA reduces effective interference of sBSs Moreover, the results indicate that by the deployment of RFA, the network significantly improves coverage either in presence or absence of DJs.

The chance of coverage probabilities in $A_{\mathrm{M}}^{o}$ versus various values of SIR threshold $\kappa_{\mathrm{M}}$ and $\varphi_{J}$ are compared in Fig. 3 .It is generated for $\varphi_{J}=0,5,10,15,20,25$. This figure shows by deploying RFA, coverage improves. This is because of efficient use of resource and effective reduction of interference 


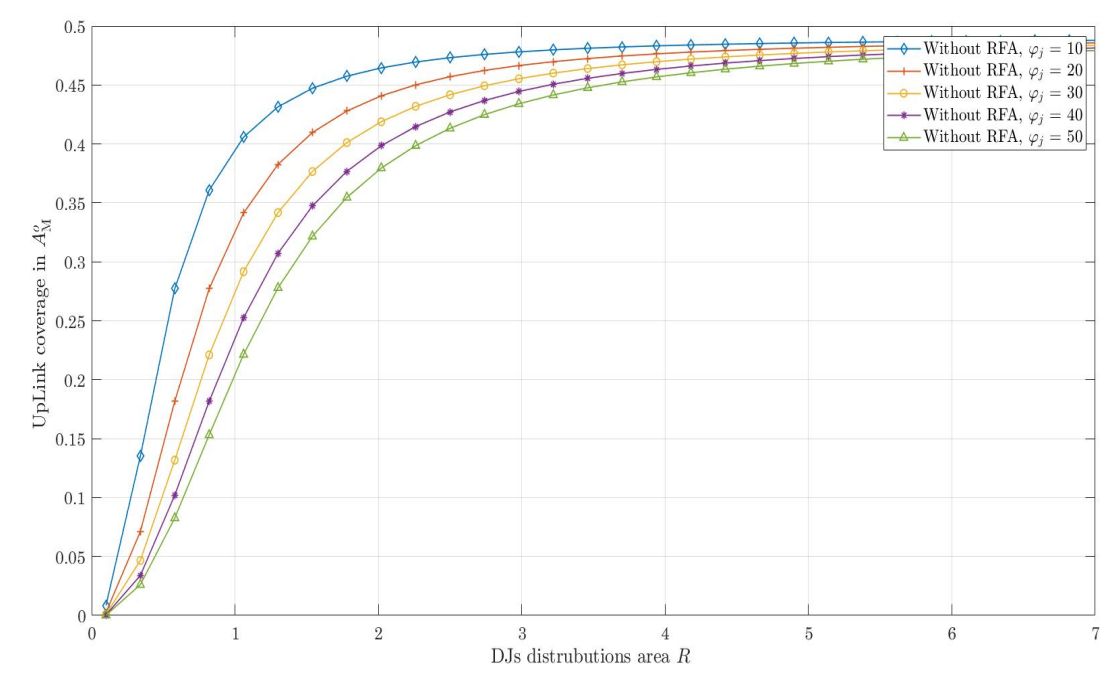

Figure 5. Without using RFA to cover DJ's distribution region,the Uplink coverage against radius .

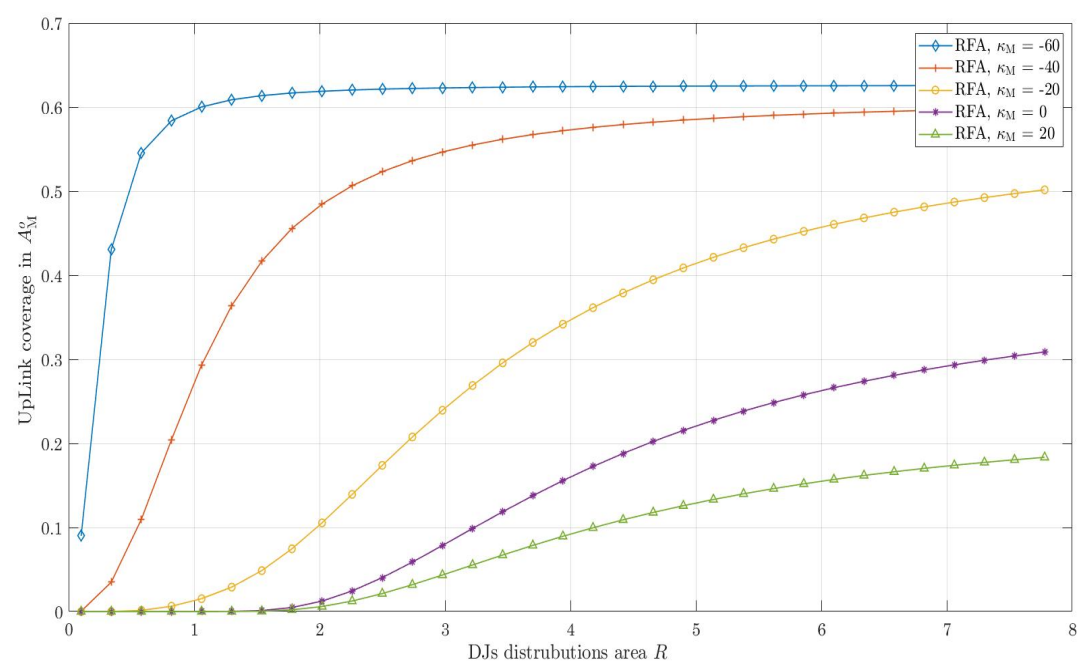

Figure 6. DJs distribution area, with the deployment of RFA, the UL coverage versus radius

due to RFA. By improving the value of $\varphi_{J}$ UL coverage severely degrades because of significant increase in DJS-I.

The coverage probabilities of UL in $A_{\mathrm{M}}$ versus DJs distribution area,considering $\kappa_{\mathrm{M}}=-40 \mathrm{~dB}$ and $\varphi_{J}=10,20,30,40$ and 50,have been shown in Figs. 4 and 5.The result indicates Improved UL coverage by increasing distribution area of DJs because of less DJs number per unit area. Thus, creating the DJs less efficient while considering their power transmission constant. Moreover, by efficient resource allocation with the employment of RFA, hence improves the UL coverage.

Similarly, the coverage probabilities of UL in $A_{\mathrm{M}}^{o}$ against different DJs distribution area radius, considering $\varphi_{J}=60$ and $\kappa_{\mathrm{M}}=-60,-40,0$ and $20 \mathrm{~dB}$ have been shown in the Figs. 6 and 7. The result shows improved UL coverage because of lower DJs-I and increased in distribution areas of $\varphi_{J}$. Hence the results indicate higher value of $\kappa_{\mathrm{M}}$ significantly reduces UL coverage because of lower user associations. 


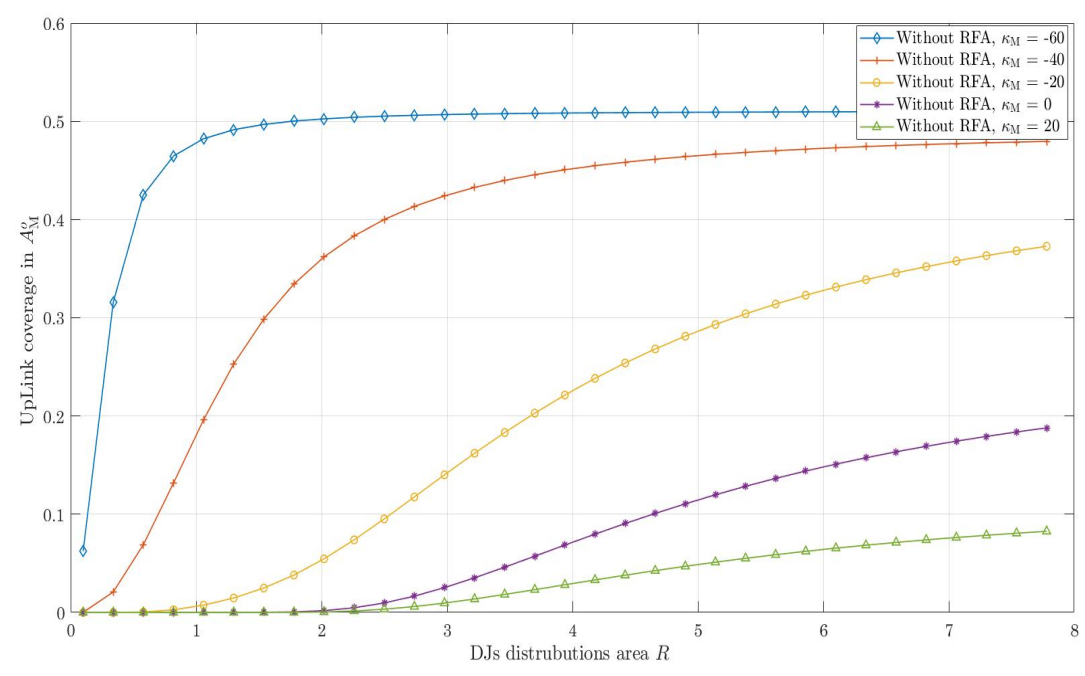

Figure 7. DJs distribution area, without RFA, the UL coverage versus radius

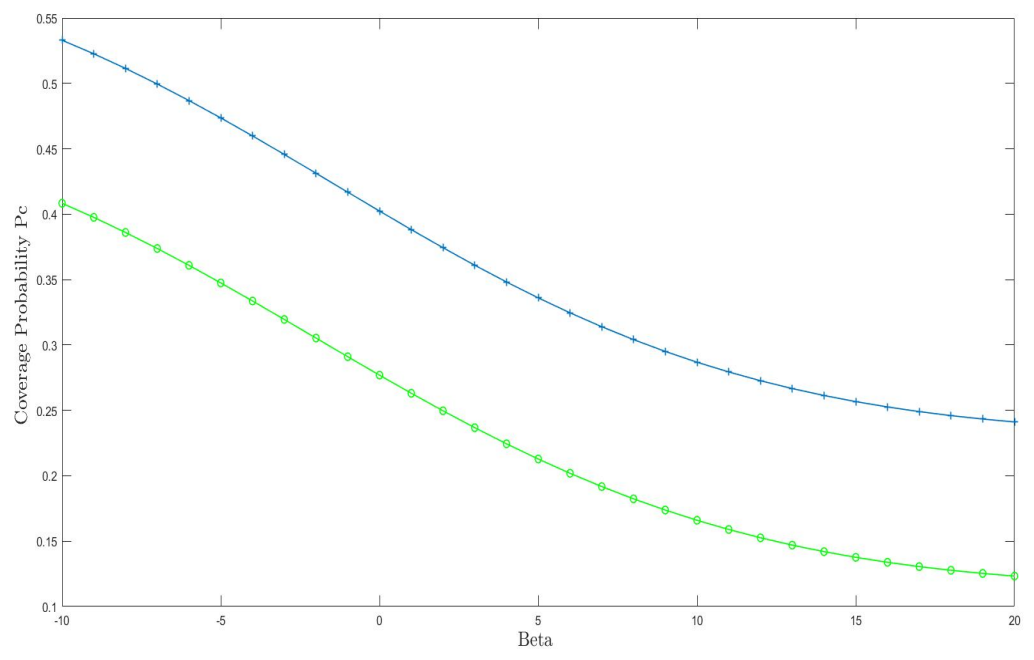

Figure 8. Path loss exponent vs Coverage Probability.

The $\beta$ is the path loss exponent while PC is the coverage probability. The Figs. 8 represents as the path loss exponent increases the coverage probability decreases while when the value of path loss decreases the coverage probability increases.

\section{Conclusion}

In HetNets ICI is the main element that restrict system recital. The situation worsens because of existence of deliberate jamming. In this paper deliberate jammers are placed around the vicinity of target. The results are originated by scrutinizing disparate network parameters including user transmit power ,radius of jammers, transmitter power of sBS and mBS and SINR threshold in case of deploying RFA and not deploying RFA. The results suggest that UL coverage is notably lessen by deliberate jammers density and transmission power. The results indicate a substantial improvement in UL coverage by applying RFA.

\section{Appendix A Proof of the Laplace Transform of (4)}

Proof of (4): 
Table 3. The Parameters of Simulation

\begin{tabular}{ll}
\hline Parameters & Configuration \\
\hline mBS, sBSs, DJs & IHPPPs \\
Channel bandwidth & $10 \mathrm{MHz}$ \\
Code iterations & 1000 \\
$\varphi_{\mathrm{S}}$ & $20 / \pi(2000 \mathrm{~m})^{2}$ \\
$\varphi_{\mathrm{M}}$ & $4 / \pi(2000 \mathrm{~m})^{2}$ \\
$\varphi_{J}$ & $20 / \pi(200 \mathrm{~m})^{2}$ \\
$A$ & $\pi \times(2000 \mathrm{~m})^{2}$ \\
The powers transmitted by mBS, & $35 \mathrm{dBm}, 30 \mathrm{dBm}, 25 \mathrm{dBm}$, \\
sBS, $v$, and DJs & and $20 \mathrm{dBm}$ \\
\hline
\end{tabular}

From MBS-tier the LT of interference received, $\mathcal{L}_{I_{\mathrm{M}, A}}(s)$, in $A$, is given below

$$
\begin{aligned}
& \left.\mathcal{L}_{I_{\mathrm{M}, A}}(s) \stackrel{(a)}{=} \mathrm{E}_{I_{\mathrm{M}, A}}\left[\exp \left(-I_{\mathrm{M}, A} s\right)\right]\right|_{s=\frac{r_{\mathrm{M}}^{\beta} \kappa_{\mathrm{M}}}{P_{t, v}^{\mathrm{UL}}}} \\
& \stackrel{(b)}{=} \mathrm{E}_{I_{\mathrm{M}, A},\left|h_{l}\right|^{2}}\left[\exp \left(-s \sum_{l \in \phi_{\mathrm{M}}} P_{t, \mathrm{M}}\left|h_{l}\right|^{2} r_{l}^{-\beta}\right)\right] \\
& \stackrel{(c)}{=} \mathrm{E}_{I_{\mathrm{M}, A},\left|h_{l}\right|^{2}}\left[\prod_{l \in \phi_{\mathrm{M}}} \exp \left(-\left|h_{l}\right|^{2} \gamma_{\circ} \kappa_{\mathrm{M}^{r}} r_{\mathrm{M}}^{\beta} r_{l}^{-\beta}\right)\right] \\
& \stackrel{(d)}{=} \mathrm{E}_{I_{\mathrm{M}, A}}\left[\prod_{l \in \phi_{\mathrm{M}}} \mathrm{E}_{\left|h_{l}\right|^{2}} \exp \left(-\left|h_{l}\right|^{2} \gamma_{\circ} \kappa_{\mathrm{M}} r_{\mathrm{M}^{\beta}}^{\beta} r_{l}^{-\beta}\right)\right] \\
& \stackrel{(e)}{=} \mathrm{E}_{\mathrm{M}_{\mathrm{M}, A}}\left[\prod_{l \in \phi_{\mathrm{M}}} \frac{1}{1+\gamma_{\circ} \kappa_{\mathrm{M}}\left(\frac{r_{l}}{r_{\mathrm{M}}}\right)^{-\beta}}\right] \\
& \stackrel{(f)}{=} \exp \left(-2 \pi \varphi_{\mathrm{M}} \int_{y}^{d 2} \frac{r_{l} d r_{l}}{1+\left(\frac{r_{l}}{\left(\gamma_{\circ} \kappa_{\mathrm{M}}\right)^{1 / \beta} r_{\mathrm{M}}}\right)^{\beta}}\right) \\
& \left.\stackrel{(g)}{=} \exp \left(-\pi \varphi_{\mathrm{M}}\left(\gamma_{\circ} \kappa_{\mathrm{M}}\right)^{2 / \beta} r_{\mathrm{M}}^{2} \int_{\left(\frac{d_{2}}{\gamma_{\circ} \kappa_{\mathrm{M}}^{1 / \beta} r_{\mathrm{M}}}\right)^{2}}^{\frac{d u}{\left(\gamma_{\circ} \kappa_{\mathrm{M}}\right)^{1 / \beta} r_{\mathrm{M}}}}\right)^{2 \frac{d u)^{\beta / 2}}{1+\left(\gamma^{1 / 2}\right.}}\right)
\end{aligned}
$$

The step $(a)$ is acquired by defination of Laplace Transform [24], step $(b)$ is derived from putting the value of $I_{\mathrm{M}, A}=\sum_{l \in \phi_{\mathrm{M}}} P_{t, \mathrm{M}}\left|h_{l}\right|^{2} r_{l}^{-\beta}$, into step (a). Step (c) Step (c) is achieved by putting the value of $s$, Step $(e)$ is acquired from the Laplace transform with respect to $h_{j}$, of Step $(d)$ Step $(f)$ is acquired by applying the probability generating functional (PGFL) of IHPPP [19], Step ( $g$ ) is acquired from 
putting the value of $u=\left(\frac{r_{l}}{\left(\gamma_{\circ} \kappa_{\mathrm{M}}\right)^{1 / \beta} r_{\mathrm{M}}}\right)^{2}$ in step $(f)$. Finally, computing Gauss hypergeometric functions approximation of Step $(g)(4)$ is obtained .

\section{Appendix B Proof of the Laplace transform of (17)}

Proof of (17):

$$
\begin{aligned}
& \mathcal{L}_{I_{\phi_{\mathrm{M}}, A_{\mathrm{M}}^{c}}^{\mathrm{UL}}}(s)=\left.\mathrm{E}_{I_{\phi_{\mathrm{M}}, A_{\mathrm{M}}^{c}}^{\mathrm{UL}}}\left[\exp \left(-I_{\phi_{\mathrm{M}}, A_{\mathrm{M}}^{c}}^{\mathrm{UL}} s\right)\right]\right|_{s=\frac{r_{\mathrm{M}}^{\beta} \kappa_{\mathrm{M}}}{P_{t, v}^{\mathrm{UL}}}} \\
& =\mathrm{E}_{I_{\phi_{\mathrm{M}}, A_{\mathrm{M}}^{\mathrm{UL}}},\left|h_{l}\right|^{2}}\left[\exp \left(-s \sum_{l \in \phi_{\mathrm{M}}} P_{t, v}^{\mathrm{UL}}\left|h_{l}\right|^{2} r_{l}^{-\beta}\right)\right] \\
& =\mathrm{E}_{I_{\phi_{\mathrm{M}}, A_{\mathrm{M}}^{\mathrm{U}}}^{\mathrm{UL}},\left|h_{l}\right|^{2}}\left[\prod_{l \in \phi_{\mathrm{M}}} \exp \left(-\left|h_{l}\right|^{2} \kappa_{\mathrm{M}} r_{\mathrm{M}}^{\beta} r_{l}^{-\beta}\right)\right] \\
& =\mathrm{E}_{I_{\phi_{\mathrm{M}}, A_{\mathrm{M}}^{\mathrm{UL}}}}\left[\prod_{l \in \phi_{\mathrm{M}}} \mathrm{E}_{\left|h_{l}\right|^{2}} \exp \left(-\left|h_{l}\right|^{2} \kappa_{\mathrm{M}} r_{\mathrm{M}}^{\beta} r_{l}^{-\beta}\right)\right] \\
& =\mathrm{E}_{I_{\phi_{\mathrm{M}}, A_{\mathrm{M}}^{\mathrm{UL}}}}\left[\prod_{l \in \phi_{\mathrm{M}}} \frac{1}{1+\kappa_{\mathrm{M}}\left(\frac{r_{l}}{r_{\mathrm{M}}}\right)^{-\beta}}\right] \\
& =\exp \left(-2 \pi \varphi_{\mathrm{M}} \int_{y}^{d_{1}} \frac{r_{l} d r_{l}}{1+\left(\frac{r_{l}}{\kappa_{\mathrm{M}}^{1 / \beta} r_{\mathrm{M}}}\right)^{\beta}}\right)
\end{aligned}
$$

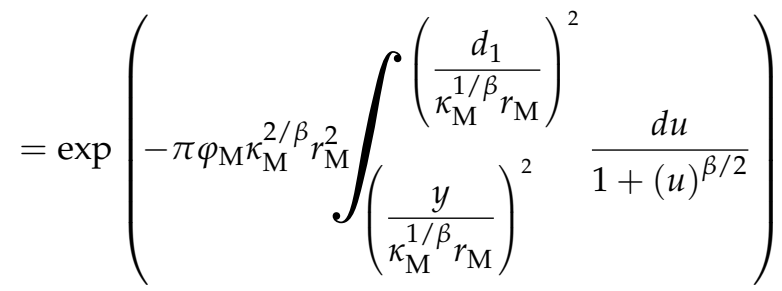

Finally, by computing the Gauss hypergeometric approximation of Step $(f)$ we arrive at (17) .

\section{Refrences}

1. Da Costa, D.B.; Duong, T.Q.; Imran, M.A.; Ngo, H.Q.; Yang, N.; Dobre, O.A. Modeling, analysis, and design of 5G ultra-dense networks. IEEE Access 2019, 7, 18894-18898.

2. Soret, B.; De Domenico, A.; Bazzi, S.; Mahmood, N.H.; Pedersen, K.I. Interference coordination for 5G new radio. IEEE Wireless Communications 2017, 25, 131-137.

3. Zou, S.; Liu, N.; Pan, Z.; You, X. Joint power and resource allocation for non-uniform topologies in heterogeneous networks. proc. 83rd Vehicular Technology Conference (VTC Spring). IEEE, 2016, pp. 1-5. 
4. Han, T.; Gong, J.; Liu, X.; Islam, S.R.; Li, Q.; Bai, Z.; Kwak, K.S. On downlink NOMA in heterogeneous networks with non-uniform small cell deployment. IEEE Access 2018, 6, 31099-31109.

5. Haroon, M.S.; Abbas, Z.H.; Abbas, G.; Muhammad, F. Coverage analysis of ultra-dense heterogeneous cellular networks with interference management. Wireless Networks 2019, pp. 1-13.

6. Hashima, S.; Muta, O.; Alghonimey, M.; Shalaby, H.; Frukawa, H.; Elnoubi, S.; Mahmoud, I. Area spectral efficiency performance comparison of downlink fractional frequency reuse schemes for MIMO heterogeneous networks. proc. International Conference on Information Science, Electronics and Electrical Engineering. IEEE, 2014, Vol. 2, pp. 1005-1010.

7. Lichtman, M.; Poston, J.D.; Amuru, S.; Shahriar, C.; Clancy, T.C.; Buehrer, R.M.; Reed, J.H. A communications jamming taxonomy. IEEE Security and Privacy 2016, 14, 47-54.

8. Muhammad Mussawer Pervez, Ziaul Haq Abbas, F.M.L.J. Location-based coverage and capacity analysis of a two tier HetNet. IET Communications 2016, 11, 1067-1073.

9. Poongup Lee, Taeyoung Lee, J.J.J.S. Interference management in LTE femtocell systems using Fractional Frequency Reuse. The 12th International Conference on Advanced Communication Technology (ICACT)Gangwon, Korea (South). IEEE, 2010, pp. 1047-1051.

10. Ziaul Haq Abbas, Fazal Muhammad, L.J. Analysis of load balancing and interference management in heterogeneous cellular networks. IEEE Access 2017, 5, 14690-14705.

11. Zhang, L.; Restuccia, F.; Melodia, T.; Pudlewski, S.M. Jam sessions: Analysis and experimental evaluation of advanced jamming attacks in MIMO networks. Proc. of the Twentieth ACM International Symposium on Mobile Ad Hoc Networking and Computing, 2019, pp. 61-70.

12. Wang, S.; Gao, Y.; Sha, N.; Zhang, G.; Zang, G. Physical layer security in K-tier heterogeneous cellular networks over nakagami-m channel during uplink and downlink phases. IEEE Access 2019, 7, 14581-14592.

13. Dong-Hee Kim, Jae-Young Ahn, H.J.K. Downlink Transmit Power Allocation in Soft Fractional Frequency Reuse Systems. ETRI Journal 2011, 33.

14. Muhammad Sajid Haroon, F.M.; Abbas, G.; Abbas, Z.H.; Hassan, A.K.; Waqas, M.; Kim, S. Interference management in ultra-dense $5 \mathrm{G}$ networks with excessive drone usage. IEEE Access 2020, 8, 102155-102164.

15. Muhammad, F.; Haroon, M.S.; Abbas, Z.H.; Abbas, G.; Kim, S. Uplink interference management for HetNets stressed by clustered wide-band jammers. IEEE Access 2019, 7, 182679-182690.

16. Kanika Grover, Alvin Lim, Q.Y. Jamming and anti-jamming techniques in wireless networks: a survey. International Journal of Ad Hoc and Ubiquitous Computing, 2013, Vol. 17, pp. 197-2015.

17. Ijaz, A.; Hassan, S.A.; Zaidi, S.A.R.; Jayakody, D.N.K.; Zaidi, S.M.H. Coverage and rate analysis for downlink HetNets using modified reverse frequency allocation scheme. IEEE Access 2017, 5, 2489-2502.

18. Fazal Muhammad, Ziaul Haq Abbas, F.Y.L. Cell association with load balancing in nonuniform heterogeneous cellular networks: Coverage probability and rate analysis. IEEE Transactions on Vehicular Technology 2016, 66, 5241-5255.

19. Jiang, X.; Zheng, B.; Zhu, W.P.; Wang, L.; Zou, Y. Large system analysis of heterogeneous cellular networks with interference alignment. IEEE Access 2018, 6, 8148-8160.

20. Haroon, M.S.; Abbas, Z.H.; Muhammad, F.; Abbas, G. Coverage analysis of cell-edge users in heterogeneous wireless networks using Stienen's model and RFA scheme. International Journal of Communication Systems, p. e4147.

21. Ponnu Jacob, A.J.; Madhukumar, A. Downlink capacity improvement and interference reduction through reverse frequency allocation. 2012 IEEE International Conference on Communication Systems (ICCS), 2012, pp. 329-333.

22. Qureshi, M.A.S.W.K.N.M.S.H.S. Clustered jamming in aerial HetNets with decoupled access. IEEE Access 2020, 8, 142218-142228.

23. Muhammad Qasim, Muhammad Sajid Haroon, M.I.F.M.S.K. 5G cellular networks: Coverage analysis in the presence of inter-cell interference and intentional jammers. Electronics 2020, 9, 1538.

24. Błaszczyszyn, B.; Haenggi, M.; Keeler, P.; Mukherjee, S. Stochastic geometry analysis of cellular networks; Cambridge University Press, 2018.

25. Haenggi, M. Stochastic geometry for wireless networks; Cambridge University Press, 2012. 
